\title{
Distinct prognostic value of different portal hypertension- associated features in patients with primary biliary cholangitis
}

\author{
Lukas Burghart ${ }^{1,2,3} \cdot$ Emina Halilbasic $^{1,3} \cdot$ Philipp Schwabl $^{1,2,4}$. \\ Benedikt Simbrunner $r^{1,2,4,5,6}$ - Albert Friedrich Stättermayer ${ }^{1,3}$. \\ Oleksandr Petrenko ${ }^{1,3,4,5,6} \cdot$ Bernhard Scheiner $^{1,2,3} \cdot$ David Bauer $^{1,2,4}$. \\ Matthias Pinter ${ }^{1,2,3} \cdot$ Kaan Boztug ${ }^{1,3,5,6}$ - Mattias Mandorfer ${ }^{1,2,3}$. \\ Michael Trauner ${ }^{1,3} \cdot$ Thomas Reiberger $^{1,2,3,4,5,6}$
}

Received: 5 June 2021/Accepted: 26 November 2021/Published online: 11 December 2021

(C) The Author(s) 2021

\begin{abstract}
Background Primary biliary cholangitis (PBC) may progress to cirrhosis and clinically significant portal hypertension (CSPH). This study assesses different features of CSPH and their distinct prognostic impact regarding decompensation and survival in patients with PBC.

Methods Patients with $\mathrm{PBC}$ were identified during a database query of our digital patient reporting system.
\end{abstract}

Thomas Reiberger

thomas.reiberger@meduniwien.ac.at

Lukas Burghart

burghartlukas@gmail.com

Emina Halilbasic

emina.halilbasic@meduniwien.ac.at

Philipp Schwabl

philipp.schwabl@meduniwien.ac.at

Benedikt Simbrunner

benedikt.simbrunner@meduniwien.ac.at

Albert Friedrich Stättermayer

albertfriedrich.staettermayer@meduniwien.ac.at

Oleksandr Petrenko

oleksandr.petrenko@meduniwien.ac.at

Bernhard Scheiner

bernhard.scheiner@meduniwien.ac.at

David Bauer

david.bauer@meduniwien.ac.at

Matthias Pinter

matthias.pinter@meduniwien.ac.at

Kaan Boztug

kaan.boztug@meduniwien.ac.at

Mattias Mandorfer

mattias.mandorfer@meduniwien.ac.at
Results A total of $333 \mathrm{PBC}$ patients (mean age 54.3 years, $86.8 \%$ females, median follow-up 5.8 years) were retrospectively assessed and 127 (38.1\%) showed features of CSPH: 63 (18.9\%) developed varices, 98 (29.4\%) splenomegaly, $62(18.6 \%)$ ascites and $20(15.7 \%)$ experienced acute variceal bleeding. Splenomegaly, portosystemic collaterals and esophageal varices were associated with an increased 5-year (5Y) risk of decompensation (15.0\%, $17.8 \%$ and $20.9 \%$, respectively). Patients without advanced

Michael Trauner

michael.trauner@meduniwien.ac.at

1 Division of Gastroenterology and Hepatology, Department of Internal Medicine III, Medical University of Vienna, Spitalgasse 23, 1090 Vienna, Austria

2 Vienna Hepatic Hemodynamic Laboratory, Division of Gastroenterology and Hepatology, Deparment of Internal Medicine III, Medical University of Vienna, Vienna, Austria

3 RALID Center of the ERN Rare Liver, Vienna General Hospital and Medical University of Vienna, Vienna, Austria

4 Christian-Doppler Laboratory for Portal Hypertension and Liver Fibrosis, Medical University of Vienna, Vienna, Austria

5 Ludwig Boltzmann Institute for Rare and Undiagnosed Diseases (LBI-RUD), Vienna, Austria

6 CeMM Research Center for Molecular Medicine of the Austrian Academy of Sciences, Vienna, Austria 
chronic liver disease (ACLD) had a similar 5Y-transplant free survival (TFS) (96.6\%) compared to patients with compensated ACLD (cACLD) but without CSPH (96.9\%). On the contrary, PBC patients with cACLD and CSPH (57.4\%) or decompensated ACLD (dACLD) (36.4\%) had significantly decreased $5 \mathrm{Y}$ survival rates. The combination of $\mathrm{LSM}<15 \mathrm{kPa}$ and platelets $\geq 150 \mathrm{G} / \mathrm{L}$ indicated a negligible risk for decompensation $(5 \mathrm{Y} 0.0 \%)$ and for mortality (5Y 0.0\%). Overall, $44(13.2 \%)$ patients died, with $18(40.9 \%)$ deaths attributed to CSPH-related complications.

Conclusion In PBC, features of CSPH may occur early and indicate an increased risk for subsequent decompensation and mortality. Hence, regular screening and on-time treatment for CSPH is crucial. Combining LSM and platelets serves as a valuable preliminary assessment, as $\mathrm{LSM}<15 \mathrm{kPa}$ and platelets $\geq 150 \mathrm{G} / \mathrm{L}$ indicate an excellent long-term outcome.

Keywords Portal hypertension - PBC - CSPH · Elastography $\cdot$ Decompensation

$\begin{array}{ll}\text { Abbreviations } & \\ \text { ACLD } & \text { Advanced chronic liver disease } \\ \text { AIH } & \text { Autoimmune hepatitis } \\ \text { cACLD } & \text { Compensated ACLD } \\ \text { CSPH } & \text { Clinically significant portal hypertension } \\ \text { dACLD } & \text { Decompensated ACLD } \\ \text { EBL } & \text { Endoscopic band ligation } \\ \text { FFI } & \text { For further information } \\ \text { HRS } & \text { Hepato-renal syndrome } \\ \text { HVPG } & \text { Hepatic venous pressure gradient } \\ \text { IQR } & \text { Inter quartile range } \\ \text { LSM } & \text { Liver stiffness measurement } \\ \text { MELD- } & \text { Model of End Stage Liver Disease-Score } \\ \text { Score } & \\ \text { OLT } & \text { Orthotopic liver transplantation } \\ \text { PLT } & \text { Platelet count } \\ \text { SBP } & \text { Spontaneous bacterial peritonitis } \\ \text { SD } & \text { Standard deviation } \\ \text { TE } & \text { Transient elastography } \\ \text { TFS } & \text { Transplant free survival } \\ \text { TIPS } & \text { Transjugular intrahepatic portosystemic } \\ & \text { shunt } \\ \text { UDCA } & \text { Ursodesoxycholic acid } \\ \text { w/o } & \text { Without }\end{array}$

\section{Introduction}

Primary biliary cholangitis (PBC) is a rare cholestatic liver disease that may progress to cirrhosis [1, 2]. Previous studies reported that only a small number of patients showed clinically significant portal hypertension (CSPH) at the time of PBC diagnosis [3-5]. A recent study, however, observed that the 10 year cumulative incidence of CSPH is as high as $40 \%$ [5]. Since CSPH drives severe complications [6], such as variceal bleeding and development of ascites, it is of utmost clinical importance to screen for $\mathrm{CSPH}$, as it impacts on prognosis and causes an increased mortality in patients with cirrhosis [7].

Only a few studies have described the prevalence and the specific manifestations of CSPH in patients with $\mathrm{PBC}$ so far [5, 8-11]. However, most studies either had a small sample size or did not investigate the entire spectrum of CSPH-related features and complications. Harms et al. found that 278 patients with PBC developed CSPH out of a cohort of 3224. According to their results ascites was the most prevalent feature of CSPH, accounting for $63 \%$ $(N=175)$ of all patients diagnosed with CSPH. Esophageal varices occurred less frequently, affecting only $23 \%$ $(N=65)$ [1]. Unfortunately, data concerning further disease progression after CSPH onset was not available [1].

In terms of novel and effective PBC treatments for patients with non-response/intolerance to UDCA or with high-risk of disease progression [12], the reduction of CSPH-associated complications represents a clinically relevant endpoint for future studies [13, 14].

Hence, our study aims to investigate the prevalence and features of CSPH in patients with $\mathrm{PBC}$, to report the incidence of CSPH-related complications during follow-up and to assess the predictive value of different clinical characteristics regarding subsequent decompensation and survival.

\section{Patients and methods}

\section{Study population (Fig. 1)}

Patients presenting with suspected with $\mathrm{PBC}$ at the Vienna General Hospital were identified by a query of existing databases and considered for this study if meeting diagnostic PBC criteria [2]. All patients had elevated cholestasis parameters (gGT, AP, bilirubin) as well as positive PBC-specific serology (AMA-M2; ANA-SP100; ANA-GP210, $N=306$ ) and/or PBC-specific histologic features on liver biopsy $(N=175)$, thus fulfilling at least two out of three diagnostic criteria [2]. After excluding 34 patients due to mechanical cholestasis [2], the diagnosis of 


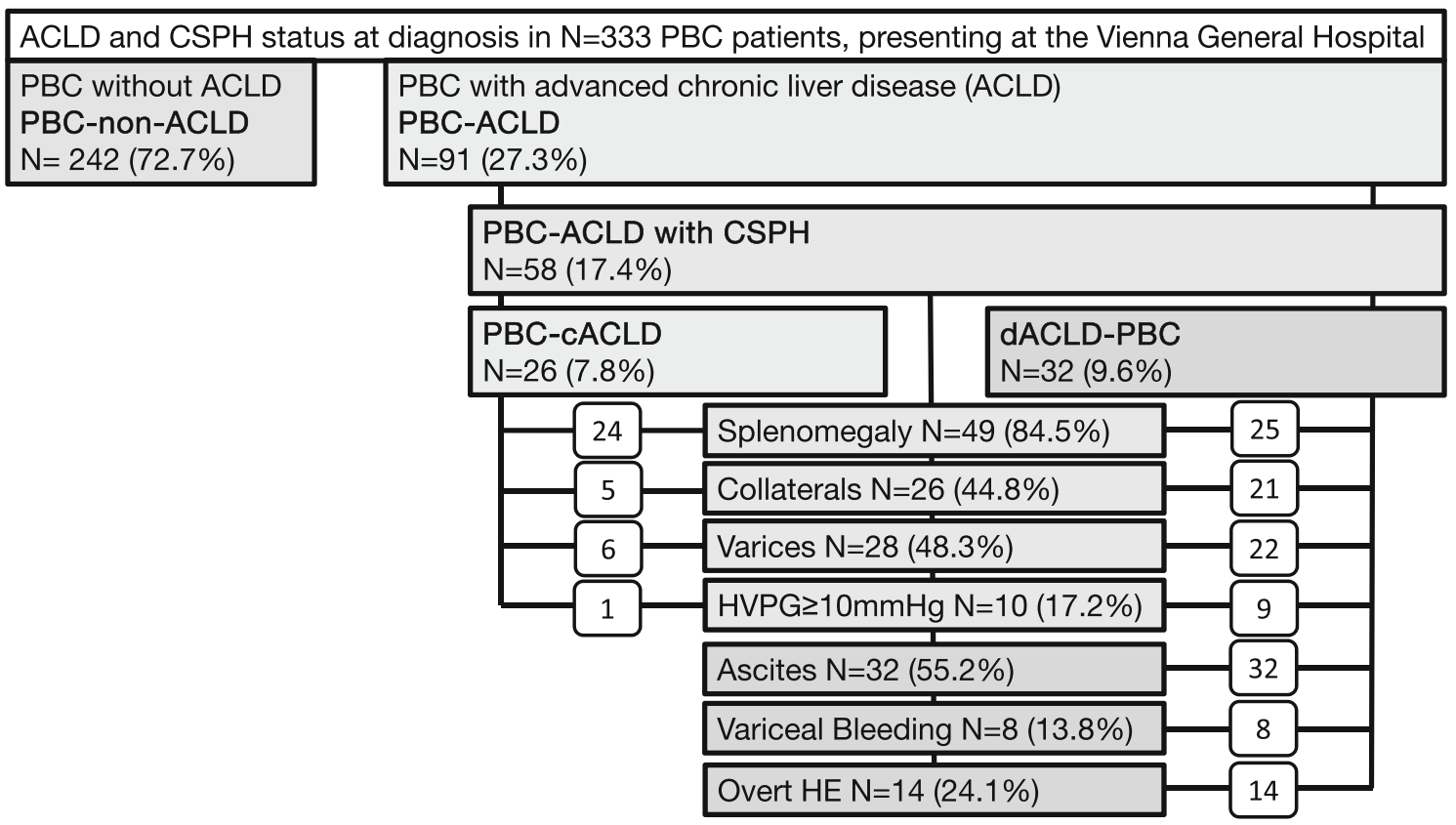

Fig. 1 Patient flowchart

PBC was confirmed by clinical documentation in a total number of $N=333$ patients.

\section{Study parameters}

Demographic data and important aspects of our patients' medical history were obtained from a database query of the electronic patient record system at the Vienna General Hospital (AKH Wien). Reports on radiologic imaging studies, such as CT, MRI and ultrasound were searched for portosystemic collaterals, portal vein thrombosis, features of mechanical cholestasis, biliary obstruction, such as cholelithiasis, as well as splenomegaly $(>11 \mathrm{~cm})$ [15]. Trained radiologists assessed the spleen diameter by measuring the maximum distance between the inferior and the superior pole in the respective imaging modality. Information regarding presence of gastroesophageal varices and endoscopic interventions on varices were obtained from endoscopy reports. Results of liver stiffness measurements (LSM) and of hepatic venous pressure gradient (HVPG) were accessed from the patients' electronic medical history.

\section{Definition of CSPH and compensated versus decompensated ACLD}

CSPH was defined by presence of at least one of the following criteria: (i) gastroesophageal varices, (ii) splenomegaly $>11 \mathrm{~cm}$, (iii) portosystemic collaterals; (iv) hepatic venous pressure gradient (HVPG) $\geq 10 \mathrm{mmHg}$; (v) ascites (excluding non-hepatic causes), (vi) variceal bleeding, (vii) hepatic encephalopathy and or (viii) death due to portal hypertension. We decided to include (i) gastroesophageal varices as well as (iii) portosystemic collaterals as distinct parameters for $\mathrm{CSPH}$, since they require different diagnostic modalities to be detected.

Advanced chronic liver disease was defined by at least one of the following criteria: (i) liver histology showing F3/F4 fibrosis, (ii) LSM $\geq 15 \mathrm{kPa}$, (iii) thrombocytopenia ( $<150 \mathrm{G} / \mathrm{L}$ ), (iv) $\mathrm{HVPG} \geq 6 \mathrm{mmHg}$ and/or (v) presence of CSPH-features (as described above). Patients with compensated advanced chronic liver disease (cACLD) were characterized by at least one feature of ACLD and the absence of any previous or current decompensating events [16-18]. Patients with decompensated ACLD (i.e. dACLD) presented at least one of the following characteristics: ascites, variceal bleeding, overt hepatic encephalopathy or death caused by portal hypertension [16-18]. HVPG- and LSM-measurements were performed when clinically indicated, as previously described [19-21]. Presence and size of gastroesophageal varices was recorded according to Austrian Billroth III guidelines [22].

Considering the clinical status at baseline and within the first year of follow-up we divided our population into the following groups: (i) patients without ACLD (nonALCLD), (ii) patients with compensated ACLD (cACLD) but without CSPH, (iii) patients with CACLD and CSPH, (iv) patients with decompensated ACLD (decompensation prior to or within the first year after PBC diagnosis; dACLD). Thus, PBC-CSPH patients comprised cACLD with CSPH and all dACLD patients but did not include cACLD patients without CSPH. 
Table 1 Patient characteristics

\begin{tabular}{|c|c|c|}
\hline \multicolumn{2}{|l|}{ Patients } & $N=333(100 \%)$ \\
\hline \multicolumn{2}{|l|}{ Age (median, IQR) } & $54.3(45.7-64.2)$ \\
\hline \multicolumn{2}{|l|}{ Women $[N(\%)]$} & $289(86.8 \%)$ \\
\hline \multicolumn{2}{|l|}{ Liver Biopsy $(N(\%))$} & $175(52.6 \%)$ \\
\hline \multicolumn{2}{|l|}{ AMA-M2(+) PBC } & $257(77.2 \%)$ \\
\hline \multicolumn{2}{|l|}{ PBC-ANA gp210 } & $57(17.1 \%)$ \\
\hline \multicolumn{2}{|l|}{ PBC-ANA sp100 } & $76(22.8 \%)$ \\
\hline \multicolumn{2}{|l|}{ Anti-centromere $\mathrm{AB}^{\mathrm{a}}$} & $42(25.6 \%)^{\infty}$ \\
\hline \multicolumn{2}{|l|}{ AIH-Overlap $[N(\%)]^{¥}$} & $77(23.1 \%)$ \\
\hline \multicolumn{2}{|l|}{ Pruritus } & $96(28.8 \%)$ \\
\hline \multicolumn{2}{|l|}{ MELD [mean (SD)] } & $7.9( \pm 3.0)$ \\
\hline \multicolumn{2}{|l|}{ LSM $[\text { median }(\mathrm{IQR} ; N)]^{\dagger}$} & $7.2 \mathrm{kPa}(5.5-13.2) \mathrm{kPa}$ \\
\hline \multicolumn{2}{|l|}{ HVPG $[\text { median }(\mathrm{IQR} ; N)]^{*}$} & $13 \mathrm{mmHg}(7-21 \mathrm{mmHg})$ \\
\hline \multicolumn{2}{|l|}{ ACLD at diagnosis } & $91(27.3 \%)$ \\
\hline \multicolumn{2}{|l|}{ cACLD without CSPH } & $33(9.9 \%)$ \\
\hline \multicolumn{2}{|l|}{ cACLD with CSPH } & $26(7.8 \%)$ \\
\hline \multicolumn{2}{|l|}{ dACLD (all with CSPH) } & $32(9.6 \%)$ \\
\hline \multicolumn{2}{|l|}{ Treatment with UDCA $[N(\%)]$} & $301(90.4 \%)$ \\
\hline \multicolumn{2}{|l|}{ UDCA dose [mg/kg, median (IQR)] } & $13.4(10.9-15.4)$ \\
\hline & At presentation & Overall, during follow-up \\
\hline Features of CSPH & $58 / 333(17.4 \%)$ & $127 / 333(38.1 \%)$ \\
\hline Splenomegaly & 49/333 (14.7\%) & 98/333 (29.4\%) \\
\hline Portosystemic collaterals & $26 / 333(7.8 \%)$ & $62 / 333(18.6 \%)$ \\
\hline Gastroesophageal varices & $28 / 333(8.4 \%)$ & 63/333 (18.6) \\
\hline $\mathrm{HVPG} \geq 10 \mathrm{mmHg}$ & $10 / 333(3.0 \%)$ & $22 / 333(6.6 \%)$ \\
\hline Ascites & $32 / 333(9.6 \%)$ & $62 / 333(18.6 \%)$ \\
\hline Variceal bleeding & $8 / 333(2.4 \%)$ & $20 / 333(6.0 \%)$ \\
\hline Hepatic encephalopathy & $14 / 333(4.2 \%)$ & $22 / 333(6.6 \%)$ \\
\hline Time between PBC diagnosis and CSPH (months, mea $N \pm \mathrm{SD}$ ) & $10.6 \pm 7.15$ & \\
\hline Features of $\mathrm{CSPH}$ before $\mathrm{PBC}$ diagnosis & $50 / 333(15.0 \%)$ & \\
\hline Median follow-up (years, median (IQR)) & $5.8(2.7-12.6)$ & \\
\hline CSPH-related decompensation & At presentation & Overall, during follow-up \\
\hline Any decompensating event & $32 / 58(55.2 \%)$ & $72 / 127(56.7 \%)$ \\
\hline Ascites & $32 / 58(55.2 \%)$ & $62 / 127(48.8 \%)$ \\
\hline Variceal bleeding & $8 / 58(13.8 \%)$ & $20 / 127(15.7 \%)$ \\
\hline Hepatic encephalopathy & $14 / 58(24.1 \%)$ & $22 / 127(17.3 \%)$ \\
\hline SBP & $2 / 58(3.4 \%)$ & $4 / 127(3.1 \%)$ \\
\hline HRS & $11 / 58(19.0 \%)$ & $21 / 127(16.5 \%)$ \\
\hline CSPH-treatment and outcomes & & \\
\hline NSBB therapy & $65 / 333(19.5 \%)$ & \\
\hline NSBB prescription in CSPH patients & $25 / 58(43.1 \%)$ & $55 / 127(43.3 \%)$ \\
\hline NSBB prescription in dACLD patients & $20 / 32(62.5 \%)$ & $43 / 72(60 \%)$ \\
\hline EBL therapy & $17 / 58(29.3 \%)$ & $36 / 127(28.3 \%)$ \\
\hline TIPS & $2 / 58(3.4 \%)$ & $7 / 127(5.5 \%)$ \\
\hline OLT & $8 / 58(13.8 \%)$ & $14 / 127(11.0 \%)$ \\
\hline Death & 16/58 (27.6) & $44 / 127(34.6 \%)$ \\
\hline
\end{tabular}


Table 1 continued

\begin{tabular}{lll}
\hline CSPH-related decompensation & At presentation & Overall, during follow-up \\
\hline CSPH-related death & $16 / 58(27.6 \%)$ & $18 / 127(14.1 \%)$
\end{tabular}

Abbreviations: please refer to the abbreviations section above

${ }^{\dagger}$ LSM by TE was performed in 217 patients

${ }^{*}$ HVPG measurements were performed in 35 patients

${ }^{¥}$ Patients with PBC-AIH overlap syndrome were diagnosed according to the Paris criteria [39]

${ }^{\infty}$ Anti-centromere status was available in $N=164$ patients

Calculation of the cumulative incidence of decompensation as well as of the LTX-free survival for each CSPH specific clinical feature (as outlined above) was based on the period between PBC diagnosis and decompensation/ death/LTX and whether patients were diagnosed with one of these characteristics during their course of disease or not. Importantly, patients with dACDL before or at baseline were not included into the calculation of the cumulative incidence of decompensation.

\section{Statistical analysis}

Data assessment and statistical analysis was performed using IBM SPSS 26. Kolmogorov-Smirnov test was applied to distinguish between normally and non-normally distributed datasets. Mean and standard deviation as well as median and interquartile range were used whenever appropriate. Student t-test and Mann-Whitney- $U$-Test were applied to assess statistical significance for comparison of metric variables whereas chi-square-test was used for comparison of nominal variables. Graph-pad Prism 8 was utilized to compute Kaplan-Meier plots to illustrate survival. The start of follow-up was defined as the date of presentation/diagnosis of PBC at our clinic.

Survival after decompensation was only calculated in those patients who already had their first decompensating event at baseline. Survival rates were calculated between date of PBC diagnosis and death or liver transplantation. Odds ratios were calculated to estimate the impact of potential risk factors for development of decompensation, death or liver transplantation.

\section{Results}

\section{PBC patient cohort (Table 1, Fig. 1)}

Our final study population included 333 patients. For further details concerning demographic characteristics, prevalence of ACLD and features of CSPH at presentation as well as targeted treatment of portal hypertension please refer to Table 1 and Fig. 1.
Table 2 Cumulative incidence of hepatic decompensation (i.e. dACLD) during followup according to distinct characteristics at clinical presentation

\begin{tabular}{lcccc}
\hline Variable & \multicolumn{4}{l}{ Cumulative incidence of dACLD } \\
\cline { 2 - 5 } & $1-3$ Years & 5 Years & 10 Years & $N$ total $\dagger$ \\
\hline Compensated patients (non-ACLD, cACLD) & $2.8 \%$ & $5.0 \%$ & $11.4 \%$ & $37 / 301$ \\
Normal PLT and LSM < 15 kPa & $0.0 \%$ & $0.0 \%$ & $5.2 \%$ & $6 / 160$ \\
Thrombocytopenia $(<150 \mathrm{G} / \mathrm{L}$ ) or LSM $\geq 15 \mathrm{kPa}$ & $8.7 \%$ & $16 \%$ & $27.9 \%$ & $19 / 51$ \\
No ACLD & $1.9 \%$ & $4.5 \%$ & $11.0 \%$ & $32 / 242$ \\
cACLD without CSPH $^{*}$ & $4.3 \%$ & $4.3 \%$ & $21.5 \%$ & $3 / 33$ \\
cACLD with CSPH $^{\ddagger}$ & $13.3 \%$ & $13.3 \%$ & $13.3 \%$ & $2 / 26$ \\
Any CSPH (cACLD + dACLD) at baseline & $18.4 \%$ & $26.8 \%$ & $41.4 \%$ & $8 / 58$ \\
Splenomegaly & $6.3 \%$ & $15.0 \%$ & $29.9 \%$ & $24 / 73$ \\
Esophageal varices & $10.1 \%$ & $20.9 \%$ & $37.5 \%$ & $26 / 41$ \\
Portosystemic collaterals & $7.4 \%$ & $17.8 \%$ & $36.9 \%$ & $26 / 41$ \\
\hline
\end{tabular}

Risk of hepatic decompensation according to distinct clinical characteristics within 1-3 years, 5 years and 10 years of follow-up

${ }^{\dagger}$ Number of patients with at least one decompensating event/number of patients included in this analysis

TSubgroup classification according to baseline characteristics

${ }^{¥}$ Baseline decompensation events were not considered 
(A) Decompensation after PBC diagnosis/presentation

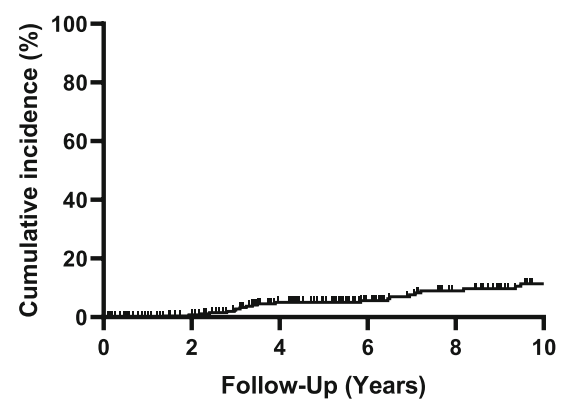

(C) Decompensation with vs. without splenomegaly $(>11 \mathrm{~cm})$

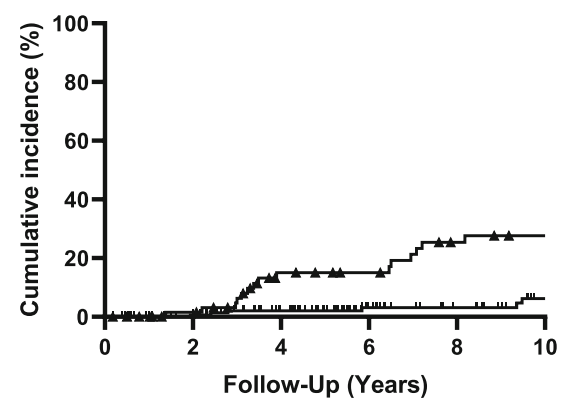

(E) Decompensation with vs. without esophageal varices

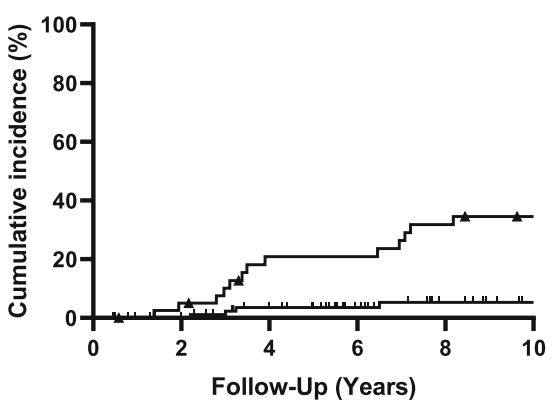

Fig. 2 Decompensation rates in PBC patients according to CSPH features*. A 10-year cumulative incidence of dACLD among all PBC patients, who were compensated at baseline; $N=21 / 301$ ( $N=$ event/total). B 10-year cumulative incidence of first or further decompensation in patients with CSPH at baseline $(N=8 / 58)(N=8 /$ 58 ; $\log$-rank $P<0.0001)$ vs. without CSPH at baseline $(N=20 / 275)$. C 10-year cumulative incidence of dACLD in compensated PBC patients with splenomegaly $(N=16 / 73$; log-rank $P<0.001)$ vs. without splenomegaly $(N=6 / 169)$. D 10-year cumulative incidence of dACLD in compensated patients with portosystemic collaterals $(N=14 / 41 ; \log$-rank $P<0.0001)$ vs. without portosystemic collaterals $(N=8 / 213)$. E 10-year cumulative incidence of dACLD in

\section{Development of ACLD and CSPH during follow-up} (Table 1, Fig. 1)

During a median follow-up duration of 5.8 (IQR 2.7-12.6) years, $168(50.5 \%)$ patients presented features suggestive of ACLD, of whom 41 (12.3\%) remained without features

(B) First/Further decompensation in patients with/without CSPH at baseline
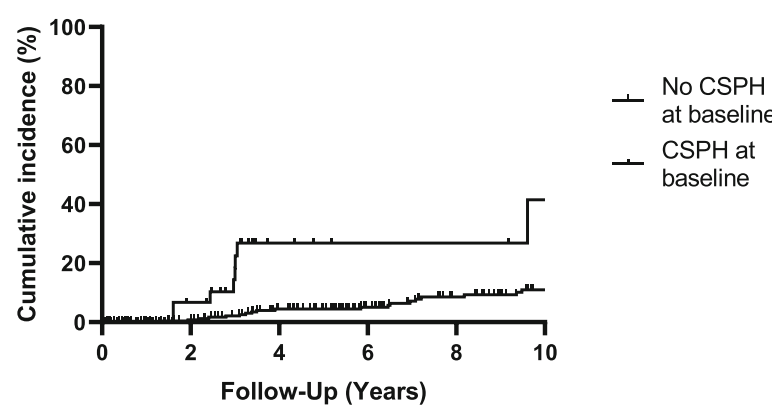

(D) Decompensation with vs. without portosystemic collaterals

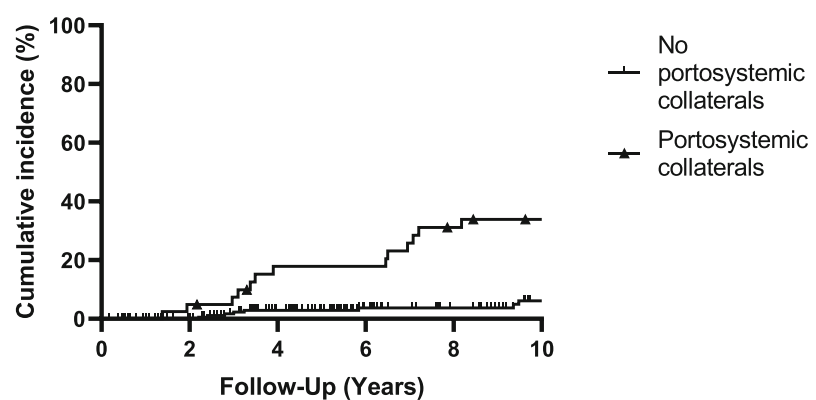

(F) Decompensation with PLT $\geq 150 \mathrm{G} / \mathrm{L}$ and $\mathrm{LSM}<15 \mathrm{kPa}$ vs. PLT $<150 \mathrm{G} / \mathrm{L}$ and/or $\mathrm{LSM} \geq 15 \mathrm{kPa}$

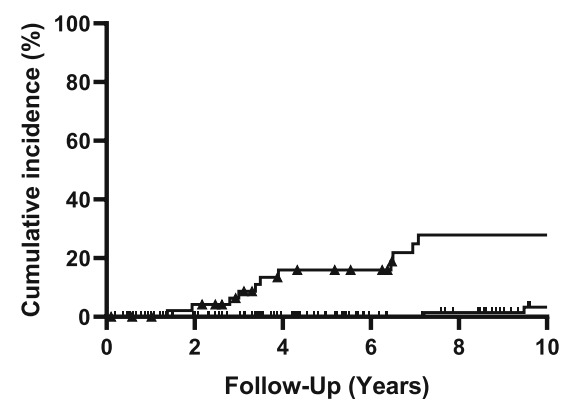

patients with esophageal varices $(N=14 / 41$; log-rank $P<0.0001)$ vs patients without esophageal varices $(N=4 / 95)$. F 10-year cumulative incidence of dACLD in patients with normal platelet count $(\geq 150 \mathrm{G} /$ L) and $\mathrm{LSM}<15 \mathrm{kPa}(N=3 / 160)$ versus patients with either thrombopenia $(<150 \mathrm{G} / \mathrm{L})$ and/or $\mathrm{LSM} \geq 15 \mathrm{kPa}(N=11 / 51$; logrank $P<0.001)$. *The number in brackets $(N=x / y)$ shows the number of patients who progressed to dACLD within 10-years $(x)$ in relation to the number of patients who met a distinct criterion (e.g., splenomegaly) and were included in this analysis $(y)$. Divergence with Table 1 is possible since we excluded patients with dACLD previous or at the time of baseline from this analysis; excluding (B)

of CSPH during further follow-up. 70 (21.0\%) developed ACLD with features of CSPH, of whom 55 (16.5\%) remained compensated throughout the observational period. All in all, $72(21.6 \%)$ patients developed dACLD, resulting in a total of $127(55+72)(38.1 \%)$ patients, identified with at least one feature of CSPH. 
Table 3 Transplant-free survival according to distinct characteristics of patients with PBC

\begin{tabular}{|c|c|c|c|c|c|}
\hline \multirow[t]{2}{*}{ Variable } & \multicolumn{5}{|c|}{ Liver transplant-free survival } \\
\hline & 1 Year & 3 Years & 5 Years & 10 Years & $\begin{array}{l}N^{\dagger} \\
\text { total }\end{array}$ \\
\hline Overall survival & $95.2 \%$ & $93.5 \%$ & $90.1 \%$ & $83.1 \%$ & $54 / 333$ \\
\hline Normal PLT count and LSM $<15 \mathrm{kPa}$ & $100.0 \%$ & $100.0 \%$ & $100.0 \%$ & $100.0 \%$ & $2 / 163$ \\
\hline Thrombocytopenia $(<150 \mathrm{G} / \mathrm{L})$ or $\mathrm{LSM} \geq 15 \mathrm{kPa}$ & $84.4 \%$ & $79.6 \%$ & $75.8 \%$ & $68.2 \%$ & $26 / 73$ \\
\hline No ACLD & $98.7 \%$ & $98.7 \%$ & $96.6 \%$ & $90.8 \%$ & $29 / 242$ \\
\hline cACLD without CSPH & $96.9 \%$ & $96.9 \%$ & $96.9 \%$ & $77.7 \%$ & $3 / 33$ \\
\hline cACLD with CSPH & $90.9 \%$ & $79.7 \%$ & $57.4 \%$ & $57.4 \%$ & $6 / 26$ \\
\hline dACLD & $69.1 \%$ & $48.5 \%$ & $36.4 \%$ & $24.3 \%$ & $16 / 32$ \\
\hline Any CSPH (cACLD + dACLD) at baseline & $79.2 \%$ & $66.3 \%$ & $47.7 \%$ & $35.8 \%$ & $22 / 58$ \\
\hline Splenomegaly & $88.4 \%$ & $83.7 \%$ & $74.2 \%$ & $67.0 \%$ & $32 / 98$ \\
\hline Portosystemic collaterals & $87.0 \%$ & $80.2 \%$ & $70.1 \%$ & $58.2 \%$ & $31 / 62$ \\
\hline Esophageal varices & $88.8 \%$ & $81.7 \%$ & $71.2 \%$ & $61.4 \%$ & $29 / 63$ \\
\hline Ascites & $84.8 \%$ & $75.4 \%$ & $66.3 \%$ & $52.6 \%$ & $29 / 62$ \\
\hline
\end{tabular}

Survival according to baseline characteristics at 1, 3, 5 and 10 years of follow-up

†Number of patients who underwent liver transplantation or died/number of patients included in this analysis
During follow-up, splenomegaly was the most frequent CSPH-related feature, affecting 98 (29.4\%) patients, whereas portosystemic collaterals were found in 62 $(18.6 \%)$ and esophageal varices in $63(18.6 \%)$ patients, respectively.

$62(18.6 \%)$ patients of our PBC cohort developed ascites, the most frequent decompensating event during follow-up. Acute variceal bleeding and hepatic encephalopathy occurred in $20(6.0 \%)$ and 22 (6.6\%) PBC patients, respectively. Overall, 18 of 127 PBC patients with CSPH (14.1\%) died due to decompensating events.

\section{Rate of CSPH-related decompensating events}

\section{(Table 2, Fig. 2)}

Among initially compensated PBC patients the 3 year decompensation rate was $2.8 \%$, whereas patients without ACLD at diagnosis showed a $1.9 \% 3$ year decompensation rate. Contrastingly, patients with cACLD but without CSPH and CACLD with CSPH had 3 year decompensation rates of $4.3 \%$ and $13.3 \%$, respectively.

Esophageal varices during gastroscopy and portosystemic collaterals on radiographic imaging showed similarly high 3 and 10 year decompensation-rates $(10.1 \%$ and $37.5 \%$ vs. $7.4 \%$ and $36.9 \%$ respectively), whereas splenomegaly revealed a comparably low 3 and 10 year decompensation rate $(6.3 \%$ and $29.9 \%)$.

\section{Survival of PBC patients according to CSPH features (Table 3, Figs. 3, 4)}

During a median follow-up of 5.8 years (IQR 2.7-12.6 years), 44 (13.21\%) PBC patients died, including 23 liver-related deaths of which 18 were attributed to CSPH-related complications. PBC patients with CSPH features had a liver related mortality of $17.3 \%$ (22/127) and a 7.2-fold risk of death compared to those without CSPH.

10 year survival of our entire PBC cohort was $83.1 \%$. PBC patients without ACLD had similar survival rates at 1, 3 and 5 years, when compared to PBC patients with cACLD without CSPH (log-rank $P=0.384$ ) (FFI Table 3, Figs. 3, 4).

Survival rates in patients with cACLD and CSPH were significantly worse when compared to patients without ACLD (log-rank $P<0.001$ ) or to patients with cACLD without CSPH (log rank $P=0.025)$. In comparison to other stages of ACLD, patients with dACLD showed the lowest one year and 10 year survival rates $(69.9 \%$ and $24.3 \%$, respectively; log-rank.

$P \leq 0.019$ ).

Patients diagnosed with splenomegaly had similar oneyear survival rates compared to patients with esophageal varices or portosystemic collaterals (FFI Table 3, Fig. 4), whereas 10 year survival rates ranged from $67.0 \%$ to $61.4 \%$ and $58.2 \%$, respectively. Patients diagnosed with ascites had the poorest one-year and 10 year survival rates (84.8\% and $52.6 \%$ respectively). 
(A) Overall survival

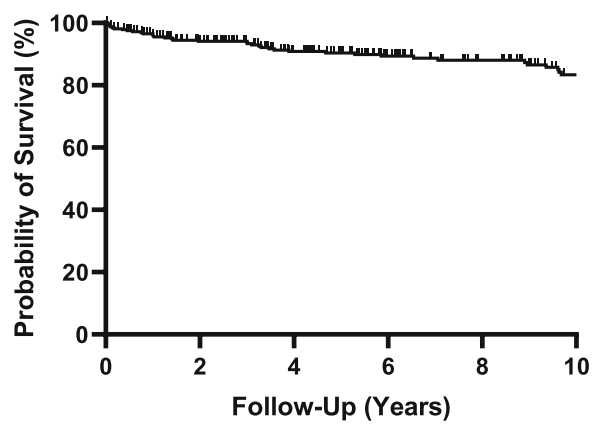

(C) Survival with vs. without portosystemic collaterals

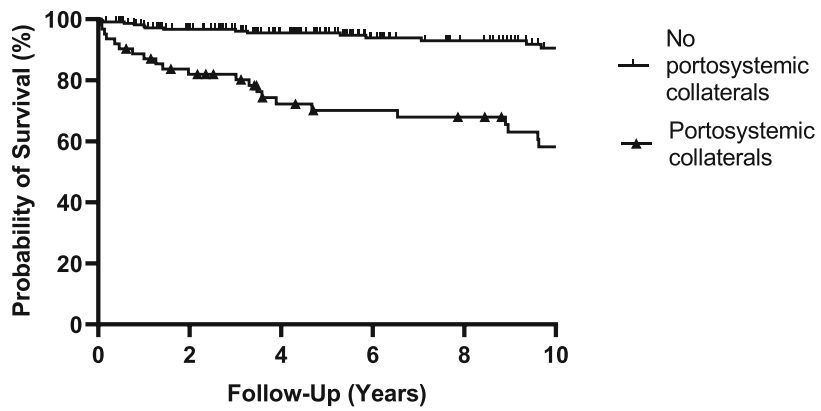

(E) Survival with vs. without ascites

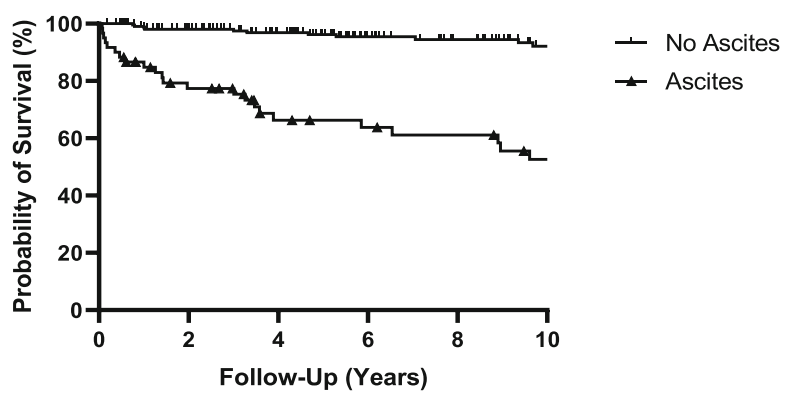

Fig. 3 LTX-free survival in PBC patients. A 10-year cumulative survival in all PBC patients $(N=38 / 333)$. B 10-year cumulative survival in patients with splenomegaly $(N=26 / 98 ;$ log-rank $P<0.0001)$ vs. without splenomegaly $(N=6 / 175)$. C 1 -year cumulative survival in patients with portosystemic collaterals $(N=22 / 62$; log-rank $P<0.0001)$ vs. without portosystemic collaterals $(N=14 /$ 224). D 10-year cumulative survival in patients with esophageal

\section{Prognostic significance of LSM and platelet count} (Tables 2, 3, Fig. 3)

Among PBC patients with both normal platelet count $(\geq 150 \mathrm{G} / \mathrm{L}$ ) and $\mathrm{LSM}<15 \mathrm{kPa}$ at transient elastography $(N=160)$, the 10 year cumulative decompensation rate was only $5.2 \%$. In contrast, patients presenting with either thrombocytopenia $(<150 \mathrm{G} / \mathrm{L})$ or $\mathrm{LSM} \geq 15 \mathrm{kPa}$ at baseline $(N=39)$ had a cumulative decompensation rate of $8.7 \%$ after 3 years and of $27.9 \%$ after 10 years of followup.
(B) Survival with vs. without splenomegaly $(>11 \mathrm{~cm})$

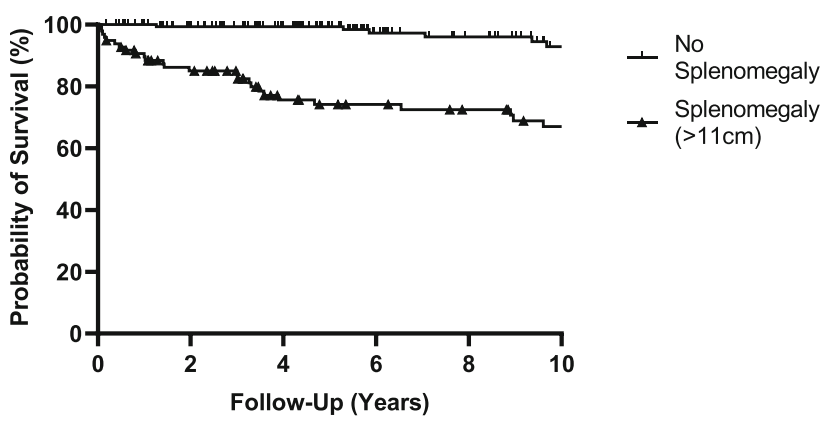

(D) Survival with vs. without esophageal varices

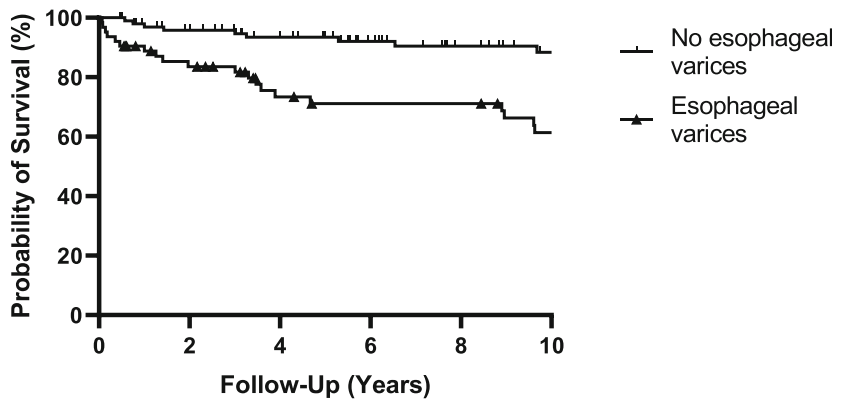

(F) Survival with PLT $\geq 150 \mathrm{G} / \mathrm{L}$ and $\mathrm{LSM}<15 \mathrm{kPa}$ vs. $P L T<150 \mathrm{G} / \mathrm{L}$ and/or $L S M \geq 15 \mathrm{kPa}$

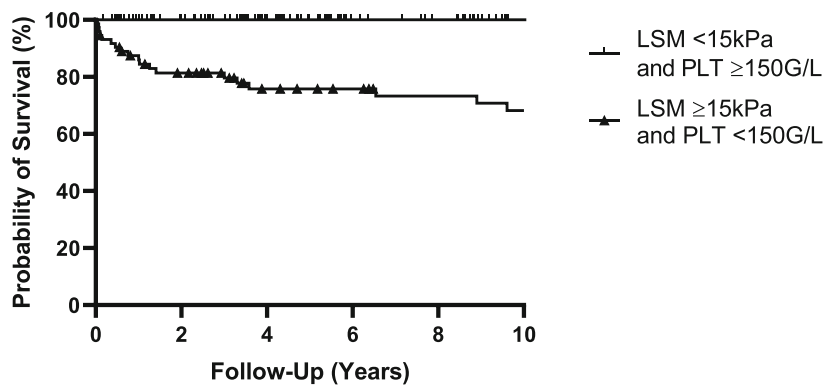

varices $(N=20 / 63$; log-rank $P<0.0001)$ vs. without esophageal varices $(N=9 / 101)$. E 10-year cumulative survival in patients with ascites $(N=23 / 62 ; \log$-rank $P<0.0001)$ vs. without ascites $(N=11$ / 217). F 10-year cumulative survival in patients with normal platelet count $(\geq 150 \mathrm{G} / \mathrm{L})$ and $\mathrm{LSM}<15 \mathrm{kPa}(N=0 / 163)$ and patients with thrombocytopenia $(<150 \mathrm{G} / \mathrm{L})$ and/or $\mathrm{LSM} \geq 15 \mathrm{kPa} \quad(N=19 / 73$; log-rank $P<0.0001)$

Patients matching at least one of the criteria (thrombocytopenia $(<150 \mathrm{G} / \mathrm{L})$ or $\mathrm{LSM} \geq 15 \mathrm{kPa}$ ) had a 13.7-fold increased risk to develop CSPH during follow-up as compared to patients with a normal platelet count and $\mathrm{LSM}<15 \mathrm{kPa}$.

Patients with both a normal platelet count and LSM < $15 \mathrm{kPa}$ had a $100 \%$ survival rate after 10 years, whereas PBC patients presenting with thrombocytopenia and/or $\mathrm{LSM} \geq 15 \mathrm{kPa}$ showed a 10 year survival rate of only $68.2 \%$. 
(A) Survival with non-ACLD vs. CACLD without CSPH vs. CACLD with CSPH vs. dACLD

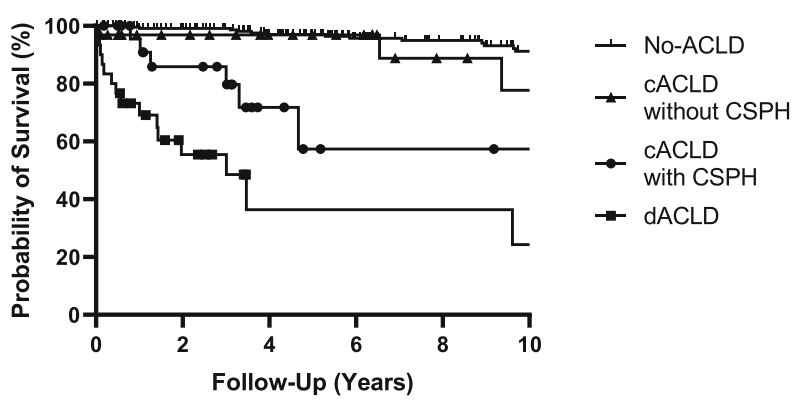

(B) Survival in patients without CSPH vs. splenomegaly vs. varices vs. portosystemic collaterals vs. ascites

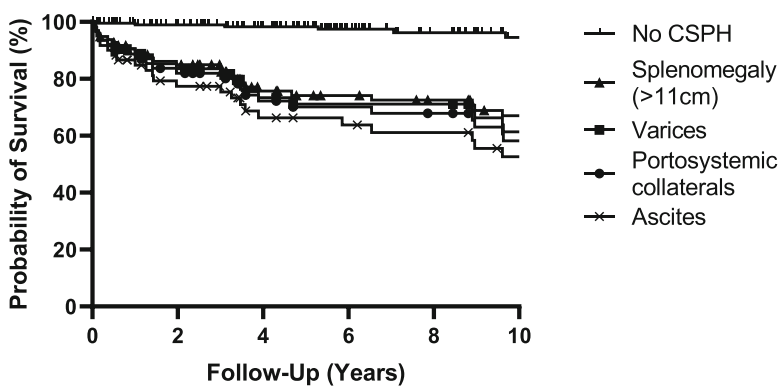

Fig. 4 LTX-free survival in PBC patients according to ACLD and CSPH status. A 10 year cumulative survival in non-ACLD $(N=14$ / $241)$ vs. CACLD without $\operatorname{CSPH}(N=3 / 33)$ vs. cACLD and CSPH $(N=6 / 26)$ vs. dACLD $(N=15 / 32)$. There was no difference in TFS between non-ACLD and cACLD w/o CSPH (log-rank $P=0.384$ ), while TFS gradually decreased in CACLD with CSPH (log-rank test vs. non-ACLD $P=0.025$ and vs. ACLD-w/o CSPH $P<0.001)$ and in dACLD (log-rank test vs. cACLD-with CSPH $P=0.019$, vs. nonACLD and cACLD-w/o CSPH $P<0.001)$. B 10-year cumulative survival in patients without CSPH $(N=7 / 205)$ vs. patients with splenomegaly $(N=26 / 98)$ vs. patients with esophageal varices $(N=20 / 63)$ vs. patients with portosystemic collaterals $(N=22 / 62)$ vs. patients with ascites $(N=23 / 62)$. There was no significant difference in TFS between splenomegaly and ascites (log-rank $P=0.0569)$ as well as between splenomegaly, portosystemic collaterals (log-rank $P=0.596$ ) and esophageal varices (log-rank $P=0.405)$. However, TFS differed significantly between patients without CSPH and splenomegaly/portosystemic collaterals/esophageal varices/ascites $(\log$-rank $P<0.0001)$

\section{Discussion}

In our PBC cohort, 10 year overall survival was $83.1 \%$ and therefore similar to recent data presented by Tanaka et al. [23] (88\% 10 year survival in UDCA treated patients).

Comparison between patients with splenomegaly, portosystemic collaterals, and esophageal varices (FFI Tables 2,3) confirms that each CSPH-related feature is linked to a different probability of subsequent decompensation and survival $[1,24]$. The same goes for stratifying patients according to their ACLD stage (FFI Tables 2,3). Hence, screening for features of CSPH in patients with PBC is crucial as it allows for early treatment intensification and individualized risk stratification.
The specific PBC target group for CSPH screening includes patients with compensated advanced chronic liver disease (cACLD), which can be suspected by LSM $\geq 15$ $\mathrm{kPa}$ [25]. The concept of cACLD, introduced at Baveno VI [25], primarily based on patients with viral hepatitis $C$, but Moctezuma-Velazquez et al. already confirmed the applicability of Baveno VI (LSM $\geq 20 \mathrm{kPa}$ and PLT $<150$ $\mathrm{G} / \mathrm{l}$ ) and extended Baveno VI criteria (LSM $\geq 25 \mathrm{kPa}$ and PLT $<150 \mathrm{G} / \mathrm{L}$ ) to predict the presence of esophageal varices requiring treatment in patients with $\mathrm{PBC}$ [26].

In this study we extended and modified the Baveno VI criteria to predict subsequent decompensation and transplant free survival. For this purpose, we decreased the LSM cutoff to $\geq 15 \mathrm{kPa}$ which is supported by recent data showing that a LSM cutoff at $\geq 14.4 \mathrm{kPa}$ has a high accuracy predicting F4 fibrosis and subsequent decompensation in patients with $\mathrm{PBC}$ [27].

Patients matching at least one of both criteria, LSM $\geq 15 \mathrm{kPa}$ and PLT $<150 \mathrm{G} / \mathrm{l}$, had a significantly increased risk for subsequent decompensation and death as opposed to those with $\mathrm{LSM}<15 \mathrm{kPa}$ and normal platelet count (FFI Tables 2, 3). This supports the use of (repeated) LSM $[20,21]$ and platelet counts in patients with PBC as both fit easily into clinical routine and hold considerable prognostic value for risk stratification.

Evaluation of PH-targeted treatment revealed that only $43.3 \%$ of those with CSPH and $60.0 \%$ of those with dACLD received NSBBs. These results indicate a significant undertreatment of CSPH in PBC patients, especially in consideration of recent studies [28], which observed a decreased risk for decompensation and mortality in CSPHpatients treated with NSBBs [29-31]. While a considerable number of PBC patients developed ascites $(N=62)$ and variceal bleeding $(N=20)$, TIPS—as a highly effective intervention to control ascites [31] and severe variceal bleeding [33] — was only used in 7 patients. Since $40.9 \%$ of all deaths observed in our PBC cohort were CSPH-related, the use of TIPS for treatment of severe CSPH complications should be encouraged, as TIPS reduces mortality in the setting of refractory ascites $[32,34]$ as well as in highrisk variceal bleeding [33,35]. Overall $N=72(21.6 \%)$ progressed to dACLD during their course of disease which confirms results from previous literature $(24.6 \%)$ [36].

$28.8 \%$ of all patients suffered from pruritus which is experienced as a significant reduction in Quality of Life [37]. However, similar to previous studies [37, 38] the odds towards development of dACLD were not increased (QR 0.86; $P=0.606$ ) in PBC patients with pruritus.

Prevalence of PBC-AIH-Overlap, which was diagnosed according to the PARIS criteria [39], was considerably higher in our cohort when compared to preexisting literature (3-10\%) [40, 41]. Recruitment of our collective of patients at the Medical University of Vienna, a tertiary care 
hospital, may have caused a selection bias towards a higher single-center prevalence of PBC-AIH Overlap Syndrome. All patients with PBC-AIH Overlap received state of the art treatment with UDCA augmented by steroids and immunosupressants [2]. Optimized therapy and regularly scheduled control visits may therefore explain why patients with AIH did not show increased odds regarding decompensation within our cohort (OR 0.937; $P=0.838$; Suppl. Table 1).

A relevant limitation of this study is its retrospective design, which impeded a comprehensive data acquisition and resulted in a heterogenous follow-up. Hence, KaplanMeier plots were used to estimate survival and the cumulative incidence of decompensation. Furthermore, selection bias towards patients with more advanced PBC disease is likely since our center is a tertiary care referral center for ACLD (of any etiology). For the same reason, we might have underestimated overall survival. Nonetheless our study population is representative for PBC patients of other tertiary care and academic centers [23]. Unfortunately, we could not address the specific impact of PBC treatment nor differentiate between UDCA responders vs. non-responders. However, as evident by the high rate of UDCA use at recommended doses, we assume that most CSPH-related complications have occurred despite UDCA therapy.

The inclusion of splenomegaly as criterion for CSPH is controversially discussed as body height and CSPH-unrelated factors such as immune dysregulation have been reported to impact on spleen size [42, 43]. Splenomegaly, however, is a widely accepted and frequent [44] clinical feature, that warrants further examination towards CSPH if detected during routine imaging. Moreover, Jung et al. [45] described splenomegaly as a "sum score" in primary sclerosing cholangitis (PSC), reflecting different pathophysiological events, including CSPH [45]. Their data additionally indicated a significantly impaired prognosis of PSC patients with increasing spleen-diameter.

We are aware that ascites and hepatic encephalopathy are not part of the initially proposed Baveno VI guidelines either. We nonetheless decided to include these parameters in our definition of CSPH since both have been associated with portal hypertension [46-48] and regularly occur during end-stage chronic liver disease.

The strength of this study is the individual assessment of different CSPH-related features, allowing clinicians to make prognostic estimations that support the planning of follow-up visits and facilitate decision-making regarding screening and treatment of CSPH-related complications. Importantly, our study confirms that risk stratification of patients with PBC according to different stages of ACLD is predictive regarding decompensation and transplant free survival. We further confirmed that the simple combination of LSM and platelet count, as a readily available score, is of excellent prognostic value in the setting of PBC. This allows for individualized care and may support early treatment intensification.

In conclusion, CSPH develops in a considerable proportion of PBC patients. Splenomegaly was the most frequent sign of CSPH and ascites the most frequent first decompensating event. The combination of LSM $\geq 15 \mathrm{kPa}$ and/or thrombocytopenia $(<150 \mathrm{G} / \mathrm{L})$ represents a valuable non-invasive risk score for CSPH-related decompensation and mortality in patients with PBC. Clinicians should regularly monitor $\mathrm{PBC}$ patients for distinct features of $\mathrm{CSPH}$, such as splenomegaly or portosystemic collaterals as they may occur earlier than varices at endoscopy but already indicate an impaired prognosis. Considering that as many as $40.9 \%$ of all deaths in our PBC cohort were caused by CSPH-related complications, the use of NSBB and TIPS for treatment of CSPH should be encouraged.

Future studies should evaluate if CSPH screening and early initiation of CSPH-targeted therapies improve prognosis and survival in patients with PBC.

Author contributions Study concept and design: LB, MM, MT, TR. Acquisition of data: LB. Analysis and interpretation of data: LB. Drafting of the manuscript: LB. Critical revision of the manuscript for important intellectual content: all authors. Statistical analysis: LB; Study supervision: TR. All authors had access to the study data and reviewed and approved the final manuscript.

Funding Open access funding provided by Medical University of Vienna. This study was not supported by funding.

Data availability Data, analytic methods, and study materials will be made available upon request by the corresponding author.

\section{Declarations}

Conflict of interest Dr Burghart, Dr Halilbasic, Dr Petrenko and Dr Boztug declare no conflict of interest. Dr Schwabl received speaking honoraria from Bristol-Myers Squibb and Boehringer-Ingelheim, consulting fees from PharmaIN and travel support from Falk. Dr Simbrunner received travel support from Abbvie and Gilead. Dr Stättermayer consults for, advises, and/or is on the speakers' bureau for Boehringer Ingelheim, Gilead, and MSD. Dr Scheiner received travel support from Abbvie, Gilead and Ipsen. Dr Bauer received travel support from Abbvie and Gilead and has received speaker fees from Abbvie. Dr Mandorfer consults for, advises, is on the speakers' bureau for, and/or received travel support from AbbVie, BristolMyers Squibb, and Gilead. He consults for, advises, and/or is on the speakers' bureau for W.L. Gore \& Associates. Dr Pinter is an investigator for Bayer, BMS, Lilly, and Roche; he received speaker honoraria from Bayer, BMS, Eisai, Lilly, and MSD; he is a consultant for Bayer, BMS, Ipsen, Eisai, Lilly, MSD, and Roche; he received travel support from Bayer and BMS. Prof Trauner received grant support from Albireo, Cymabay, Falk, Gilead, Intercept, MSD, and Takeda, honoraria for consulting from BiomX, Boehringer Ingelheim, Falk, Genfit, Gilead, Intercept, Janssen, MSD, Novartis, Phenex, and Regulus, speaker fees from BMS, Falk, Gilead, Intercept and MSD, as well as travel support from Abbvie, Falk, Gilead and Intercept. Prof Reiberger received grant support from Abbvie, Boehringer- 
Ingelheim, Gilead, MSD, Philips Healthcare, Gore; speaking honoraria from Abbvie, Gilead, Gore, Intercept, Roche, MSD; consulting/ advisory board fee from Abbvie, Bayer, Boehringer-Ingelheim, Gilead, Intercept, MSD, Siemens; and travel support from Boehringer-Ingelheim, Gilead and Roche.

Supplementary InformationThe online version contains supplementary material available at https://doi.org/10.1007/s00535021-01839-3.

Open Access This article is licensed under a Creative Commons Attribution 4.0 International License, which permits use, sharing, adaptation, distribution and reproduction in any medium or format, as long as you give appropriate credit to the original author(s) and the source, provide a link to the Creative Commons licence, and indicate if changes were made. The images or other third party material in this article are included in the article's Creative Commons licence, unless indicated otherwise in a credit line to the material. If material is not included in the article's Creative Commons licence and your intended use is not permitted by statutory regulation or exceeds the permitted use, you will need to obtain permission directly from the copyright holder. To view a copy of this licence, visit http://creativecommons. org/licenses/by/4.0/.

\section{References}

1. Harms MH, Lammers WJ, Thorburn D, et al. Major hepatic complications in ursodeoxycholic acid-treated patients with primary biliary cholangitis: risk factors and time trends in incidence and outcome. Am J Gastroenterol. 2018;113:254-64.

2. European Association for the Study of the Liver. Electronic address: easloffice@easloffice.eu, European Association for the Study of the Liver. EASL clinical practice guidelines: the diagnosis and management of patients with primary biliary cholangitis. J Hepatol. 2017;67:145-72.

3. Lammers WJ, Hirschfield GM, Corpechot C, et al. Development and validation of a scoring system to predict outcomes of patients with primary biliary cirrhosis receiving ursodeoxycholic acid therapy. Gastroenterology. 2015;149:1804-1812.e4.

4. Carbone M, Mells GF, Pells G, et al. Sex and age are determinants of the clinical phenotype of primary biliary cirrhosis and response to ursodeoxycholic acid. Gastroenterology. 2013;144:560-569. e7 (quiz e13-14).

5. Trivedi PJ, Hirschfield GM. Primary biliary cirrhosis: renaming primary biliary cirrhosis-clarity or confusion? Nat Rev Gastroenterol Hepatol. 2015;12:678-9.

6. Semmler G, Simbrunner B, Scheiner B, et al. Impact of farnesoid $\mathrm{X}$ receptor single nucleotide polymorphisms on hepatic decompensation and mortality in cirrhotic patients with portal hypertension. J Gastroenterol Hepatol. 2019;34:2164-72.

7. D'Amico G, Garcia-Tsao G, Pagliaro L. Natural history and prognostic indicators of survival in cirrhosis: a systematic review of 118 studies. J Hepatol. 2006;44:217-31.

8. Chan C-W, Tsochatzis EA, Carpenter JR, et al. Predicting the advent of ascites and other complications in primary biliary cirrhosis: a staged model approach. Aliment Pharmacol Ther. 2010;31:573-82.

9. Shi T-Y, Zhang L-N, Chen H, et al. Risk factors for hepatic decompensation in patients with primary biliary cirrhosis. World J Gastroenterol. 2013;19:1111-8.

10. Joshita S, Umemura T, Ota $M$, et al. AST/platelet ratio index associates with progression to hepatic failure and correlates with histological fibrosis stage in Japanese patients with primary biliary cirrhosis. J Hepatol. 2014;61:1443-5.
11. Patanwala I, McMeekin P, Walters R, et al. A validated clinical tool for the prediction of varices in PBC: the Newcastle Varices in PBC Score. J Hepatol. 2013;59:327-35.

12. Nevens F, Andreone P, Mazzella G, et al. A placebo-controlled trial of obeticholic acid in primary biliary cholangitis. N Engl $\mathrm{J}$ Med. 2016;375:631-43.

13. Abraldes JG, Garcia-Tsao G. The design of clinical trials in portal hypertension. Semin Liver Dis. 2017;37:73-84.

14. Abraldes JG, Tandon P. Soft and hard endpoints in acute variceal bleeding. Hepatology. 2015;61:762-5.

15. Frank K, Linhart P, Kortsik C, et al. Sonographic determination of spleen size: normal dimensions in adults with a healthy spleen. Ultraschall Med. 1986;7:134-7.

16. Bastati N, Beer L, Mandorfer M, et al. Does the functional liver imaging score derived from gadoxetic acid-enhanced MRI predict outcomes in chronic liver disease? Radiology. 2020;294:98-107.

17. Scheiner B, Steininger L, Semmler G, et al. Controlled attenuation parameter does not predict hepatic decompensation in patients with advanced chronic liver disease. Liver Int. 2019;39:127-35.

18. Simbrunner B, Marculescu R, Scheiner B, et al. Non-invasive detection of portal hypertension by enhanced liver fibrosis score in patients with different aetiologies of advanced chronic liver disease. Liver Int. 2020;40:1713-24.

19. Reiberger T, Schwabl P, Trauner M, et al. Measurement of the hepatic venous pressure gradient and transjugular liver biopsy. JoVE (J Vis Exp). 2020;160:e58819.

20. Schwabl P, Bota S, Salzl P, et al. New reliability criteria for transient elastography increase the number of accurate measurements for screening of cirrhosis and portal hypertension. Liver Int. 2015;35:381-90.

21. Reiberger T, Ferlitsch A, Payer BA, et al. Noninvasive screening for liver fibrosis and portal hypertension by transient elastography-a large single center experience. Wien Klin Wochenschr. 2012;124:395-402.

22. Reiberger T, Püspök A, Schoder M, et al. Austrian consensus guidelines on the management and treatment of portal hypertension (Billroth III). Wien Klin Wochenschr. 2017;129:135-58.

23. Tanaka A, Hirohara J, Nakano T, et al. Association of bezafibrate with transplant-free survival in patients with primary biliary cholangitis. J Hepatol. 2021;75:565-571.

24. Takeshita E, Kumagi T, Matsui H, et al. Esophagogastric varices as a prognostic factor for the determination of clinical stage in patients with primary biliary cirrhosis. J Gastroenterol. 2003;38:1060-5.

25. de Franchis R, Baveno VI Faculty. Expanding consensus in portal hypertension: report of the Baveno VI consensus workshop: stratifying risk and individualizing care for portal hypertension. J Hepatol. 2015;63:743-52.

26. Moctezuma-Velazquez C, Saffioti F, Tasayco-Huaman S, et al. Non-invasive prediction of high-risk varices in patients with primary biliary cholangitis and primary sclerosing cholangitis. Am J Gastroenterol. 2019;114:446-52.

27. Osman KT, Maselli DB, Idilman IS, et al. Liver stiffness measured by either magnetic resonance or transient elastography is associated with liver fibrosis and is an independent predictor of outcomes among patients with primary biliary cholangitis. J Clin Gastroenterol. 2020;021(55):449-57.

28. Villanueva $\mathrm{C}$, Albillos $\mathrm{A}$, Genescà $\mathrm{J}$, et al. $\beta$ blockers to prevent decompensation of cirrhosis in patients with clinically significant portal hypertension (PREDESCI): a randomised, double-blind, placebo-controlled, multicentre trial. Lancet. 2019;393:1597-608.

29. Reiberger T, Ferlitsch A, Payer BA, et al. Non-selective betablocker therapy decreases intestinal permeability and serum 
levels of LBP and IL-6 in patients with cirrhosis. J Hepatol. 2013;58:911-21.

30. Reiberger T, Ulbrich G, Ferlitsch A, et al. Carvedilol for primary prophylaxis of variceal bleeding in cirrhotic patients with haemodynamic non-response to propranolol. Gut. 2013;62:1634-41.

31. Reiberger $\mathrm{T}$, Mandorfer M. Beta adrenergic blockade and decompensated cirrhosis. J Hepatol. 2017;66:849-59.

32. Bucsics $\mathrm{T}$, Hoffman S, Grünberger J, et al. ePTFE-TIPS vs repetitive LVP plus albumin for the treatment of refractory ascites in patients with cirrhosis. Liver Int. 2018;38:1036-44.

33. Bucsics T, Schoder M, Diermayr M, et al. Transjugular intrahepatic portosystemic shunts (TIPS) for the prevention of variceal re-bleeding-a two decades experience. PLoS One. 2018;13:e0189414.

34. Bureau C, Thabut D, Oberti F, et al. Transjugular intrahepatic portosystemic shunts with covered stents increase transplant-free survival of patients with cirrhosis and recurrent ascites. Gastroenterology. 2017;152:157-63.

35. García-Pagán JC, Caca K, Bureau C, et al. Early use of TIPS in patients with cirrhosis and variceal bleeding. $\mathrm{N}$ Engl $\mathrm{J}$ Med. 2010;362:2370-9.

36. John BV, Khakoo NS, Schwartz KB, et al. Ursodeoxycholic acid response is associated with reduced mortality in primary biliary cholangitis with compensated cirrhosis. Am J Gastroenterol. 2021;116:1913-23.

37. Hirschfield GM, Dyson JK, Alexander GJM, et al. The British Society of Gastroenterology/UK-PBC primary biliary cholangitis treatment and management guidelines. Gut. 2018;67:1568-94.

38. Dickson ER, Grambsch PM, Fleming TR, et al. Prognosis in primary biliary cirrhosis: model for decision making. Hepatology. 1989;10:1-7.

39. Chazouillères $O$, Wendum D, Serfaty L, et al. Primary biliary cirrhosis-autoimmune hepatitis overlap syndrome: clinical features and response to therapy. Hepatology. 1998;28:296-301.
40. Freedman BL, Danford CJ, Patwardhan V, et al. Treatment of overlap syndromes in autoimmune liver disease: a systematic review and meta-analysis. J Clin Med. 2020;9:1449.

41. Boberg KM, Chapman RW, Hirschfield GM, et al. Overlap syndromes: the International Autoimmune Hepatitis Group (IAIHG) position statement on a controversial issue. J Hepatol. 2011;54:374-85.

42. McKenzie CV, Colonne CK, Yeo JH, et al. Splenomegaly: pathophysiological bases and therapeutic options. Int J Biochem Cell Biol. 2018;94:40-3.

43. Terayama N, Makimoto KP, Kobayashi S, et al. Pathology of the spleen in primary biliary cirrhosis: an autopsy study. Pathol Int. 1994:44:753-8.

44. Sakauchi F, Mori M, Zeniya M, et al. A cross-sectional study of primary biliary cirrhosis in Japan: utilization of clinical data when patients applied to receive public financial aid. J Epidemiol. 2005; 15:24-8.

45. Jung F, Cazzagon N, Vettorazzi E, et al. Rate of spleen length progression is a marker of outcome in patients with primary sclerosing cholangitis. Clin Gastroenterol Hepatol. 2019;17:2613-5

46. Ramanathan S, Khandelwal N, Kalra N, et al. Correlation of HVPG level with ctp score, MELD score, ascites, size of varices, and etiology in cirrhotic patients. Saudi J Gastroenterol. 2016;22:109-15.

47. Aller M-A, Arias N, Blanco-Rivero J, et al. Hepatic encephalopathy: sometimes more portal than hepatic. J Gastroenterol Hepatol. 2019;34:490-4.

48. Tapper EB, Henderson JB, Parikh ND, et al. Incidence of and risk factors for hepatic encephalopathy in a population-based cohort of Americans with cirrhosis. Hepatology Commun. 2019;3:1510-9.

Publisher's Note Springer Nature remains neutral with regard to jurisdictional claims in published maps and institutional affiliations. 